Supplemental Information

\title{
Preferential Binding of Cytochrome $c$ with Anionic Ligand-Coated Gold Nanoparticles: A Complementary Computational and Experimental Approach
}

\author{
Emily J. Tollefson ${ }^{\dagger}$, Caley R. Allen ${ }^{2}$, Gene Chong ${ }^{2}$, Xi Zhang ${ }^{3}$, Nikita D. Rozanov', Anthony \\ Bautista ${ }^{2}$, Jennifer J. Cerda ${ }^{1}$, Joel A. Pedersen ${ }^{4,5}$, Catherine J. Murphy ${ }^{3}$,Erin E. Carlson ${ }^{1 *}$, and \\ Rigoberto Hernandez ${ }^{2 *}$
}

${ }^{1}$ Department of Chemistry, University of Minnesota - Twin Cities, Minneapolis, MN 55455, United States

${ }^{2}$ Department of Chemistry, Johns Hopkins University, Baltimore, MD 21218, United States

${ }^{3}$ Department of Chemistry, University of Illinois at Urbana - Champaign, Urbana, IL 61801, United States

${ }^{4}$ Environmental Chemistry and Technology Program, University of Wisconsin - Madison, Madison, WI 53706, United States

${ }^{5}$ Department of Chemistry, University of Wisconsin - Madison, 1101 University Ave., Madison, WI 53706, United States

${ }^{\dagger}$ These authors contributed equally to the work.

* Corresponding Authors:

Rigoberto Hernandez; r.hernandez@jhu.edu

Erin Carlson; carlsone@umn.edu

Table of Contents

1. Computational Supplemental Information

A. Representative Structures of Cyt $c$ and Cyt $c$ with MPA-AuNP

B. Simulation of Cyt $c$ in Solvent

C. Relative Orientation of Cyt $c$ in Close Proximity to MPA-AuNPs

D. Cytochrome $c$ Secondary and Tertiary Structure Conformations

2. Experimental Supplemental Information
A. MPA-AuNP Characterization
B. Protein Absorption to MPA-AuNPs
C. Circular Dichroism at $\mathrm{pH} 7.0$ and $\mathrm{pH} 8.6$
D. $N$-acetoxy-succinimide Synthesis
E. MALDI-TOF Analysis of Whole Protein Labeling 

F. Footprinting Workflow
G. PEAKS Search Parameters and Summary Table

3. Additional SI Files

A. LC-MS/MS .raw files

B. Statistical Analysis Spreadsheet for Percent Modification 


\section{Computational Supplemental Information}

1A. Representative Structures of Cyt $c$ and Cyt $c$ with MPA-AuNP. The electrostatic surface maps of cytochrome $c$ (cyt $c$ ) in two particular orientations (related by a $180^{\circ}$ rotation) are shown in Fig. S1.1. The three putative cardiolipin binding sites are highlighted on the secondary structure of cyt $c$; site $\mathrm{A}$, site $\mathrm{L}$, and site $\mathrm{C}$. The specific residue identity of these sites and $\mathrm{N}$ are discussed in detail in the main text.

The starting structures for cyt $c$ relative to the NP were illustrated in the main text. Figure S1.2 supplements those representations by illustrating how cyt $c$ can be moved (without rotation) through translation to one of the six possible faces of the MPA-AuNP associated with a rectilinear lattice. In this way, the face of cyt $c$ initially facing the NP is different, and the face of the NP is also varied, and provides a simplified (albeit with a relatively small sample size) approach to sampling the possible pairwise starting structures while introducing little bias other than for the choice of the first orientation (such as the one shown in green in Fig. S1.2.)

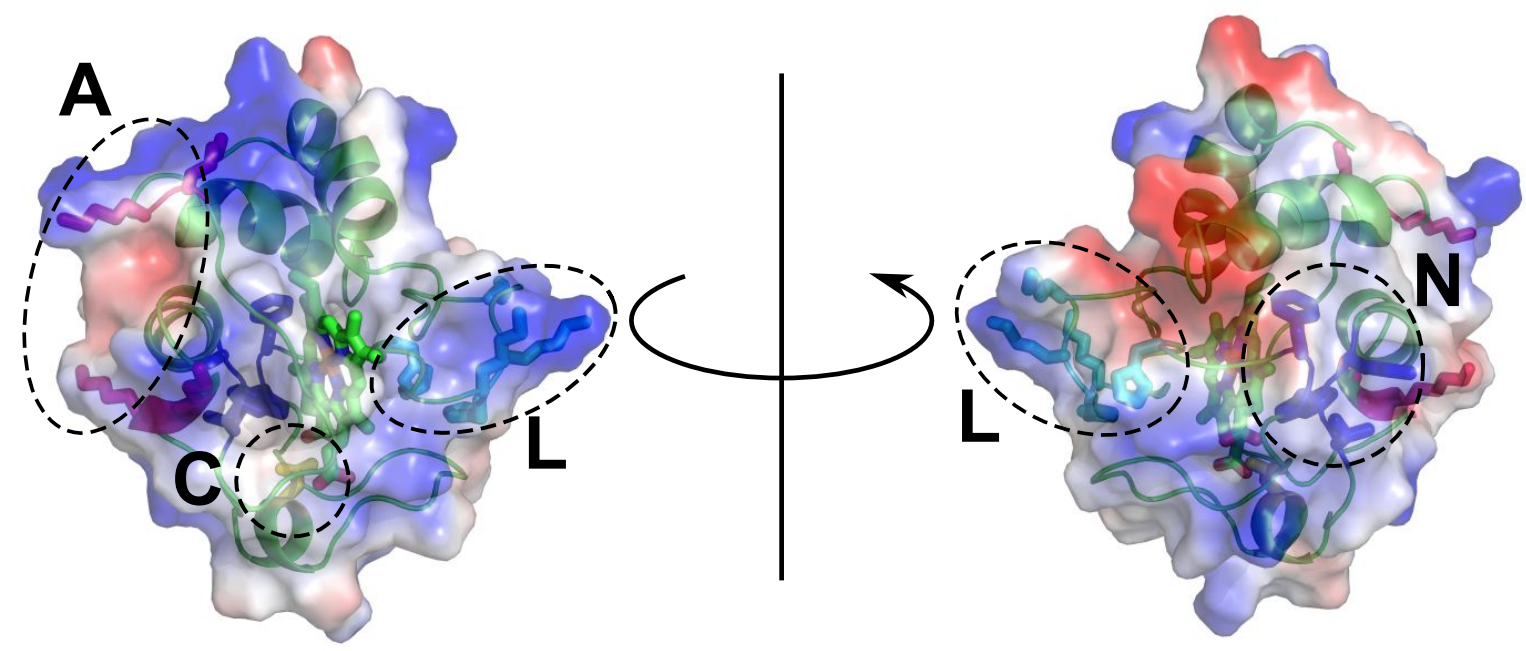

Figure S1.1. Cytochrome $c$ (cyt $c$ ) structure with the adaptive Poisson-Boltzmann electrostatic surface area maps shown on the protein. Blue indicates regions of positive charge, red indicates negative charge, and white indicates neutral regions. 


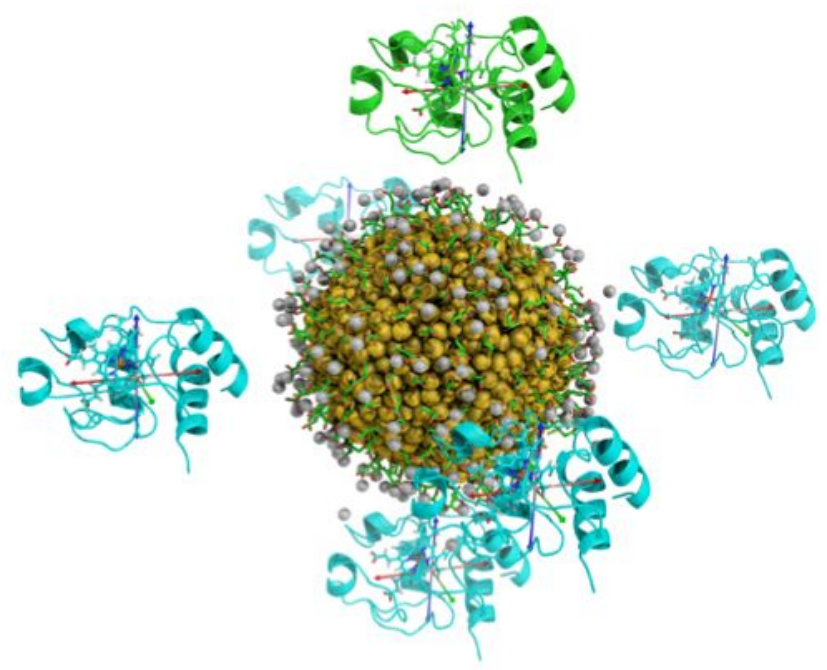

Figure S1.2. The six starting orientations used in the molecular dynamics simulations of $c y t c$ oriented around the MPA-AuNP.

1B. Simulation of $\boldsymbol{C y t} \boldsymbol{c}$ in Solvent. In order to benchmark the behavior of cyt $c$ in the simulations when not in the presence of the AuNP, a numerical control experimental was run. Namely, we prepared an equilibrated configuration with cyt $c$, but no NP present, and solvated it with TIP3P waters using the same procedures as for the cyt $c$ with MPA-AuNP systems. A 200 nanosecond trajectory was then integrated using the same protocol as that for the MPA-AuNP systems. Figure S1.3 displays the radius of gyration of the cyt $c$ over this long trajectory. The specific values at any given time are particular to the trajectory. However, the average value and degree of fluctuations are statistically relevant. As noted in the main text, the average value is consistent with the experimentally reported value of $13.6 \AA{ }^{1}$ 


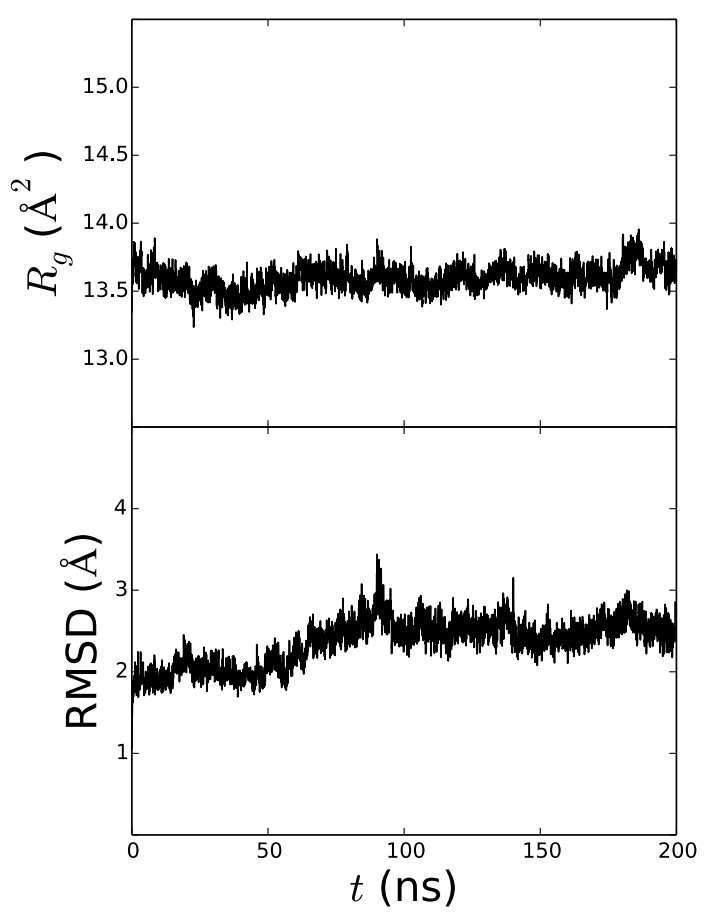

Figure S1.3. The radius of gyration $\left(\mathrm{R}_{g}\right)$ and root mean square deviation (RMSD) as a function of time for cyt $c$ in a periodic box of TIP3P water for $200 \mathrm{~ns}$. The RMSD of cyt $c$ is aligned to the final frame at the end of the $200 \mathrm{~ns}$ trajectory.

1C. Relative Orientation of Cyt $c$ in Close Proximity to MPA-AuNPs. Figure S1.4 provides the distance between the selected sites of cyt $c$ and MPA-AuNP in three trajectories starting from configurations with the three initial faces toward the nanoparticle not shown in the main text. In each of these three cases, cyt $c$ begins with a particular binding site - either site N, A or L for trajectories 1,2 or 5, respectively. In the case of trajectories 2 and 5 , cyt $c$ remains in that relative orientation for the entirety of the 200 nanosecond trajectories, as also shown in Figures S1.5 and S1.6, respectively. In the case of trajectory 1 , cyt $c$ appears to not be relaxed at the end of the trajectory and moving toward an orientation in which site $\mathrm{N}$ would not be facing the nanoparticle. Thus, these three trajectories merely indicate that the dynamic orientations with site $\mathrm{A}$ and $\mathrm{L}$ facing the MPA-AuNP are metastable up to, at least, timescales on the order of 200 nanoseconds.

1D. Cytochrome $c$ Secondary and Tertiary Structure Conformations. Figure S1.7 provides the radius of gyration for trajectories 1,2 , and 5 complementing the data provided for trajectories 3,4 , and 6 in Figure 6 of the main text.

The RMSD's of the trajectories for cyt $c$ and the heme group relative to their initial structures are shown here in Figure S1.8. As summarized in the text, they provide little new insight in the dynamics of the proteins in so far as they agree fully with the interpretation gained from analysis of the radius of gyration. 


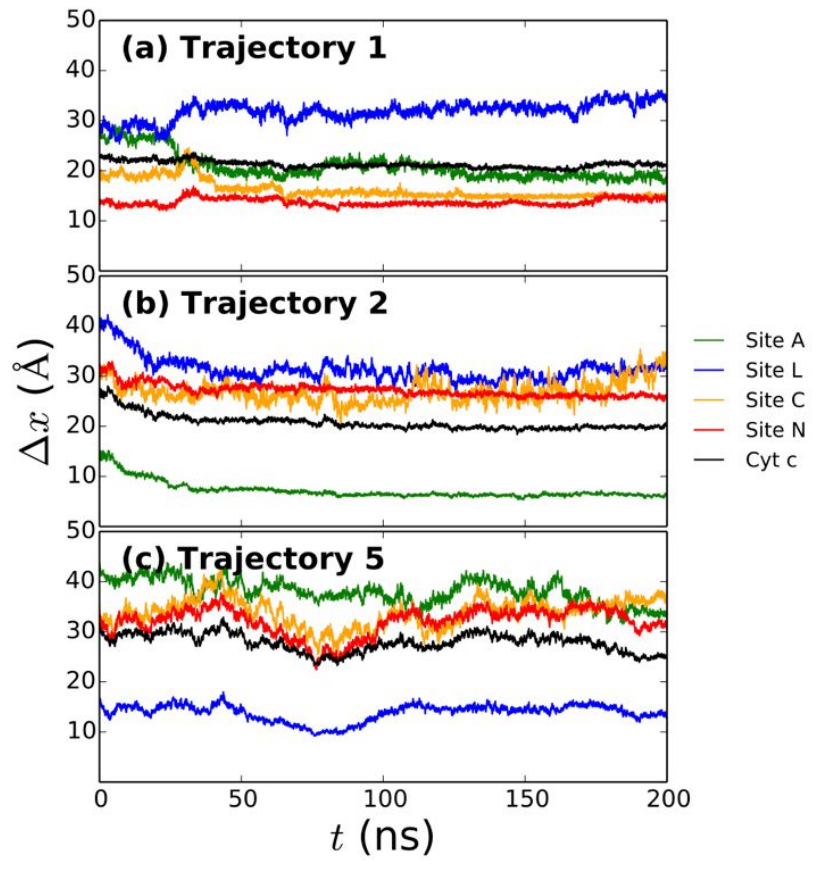

Figure S1.4. The distance $\Delta x$ between the COM positions of the cyt $c$ binding sites and the MPA-AuNP surface were monitored for trajectories 1, 2, and 5. The green, blue, orange, red, and black curves are the measured distances between the bare AuNP surface and the COM of site A, site L, site $\mathrm{C}$, and site $\mathrm{N}$ or the cyt $c$ COM, respectively. 


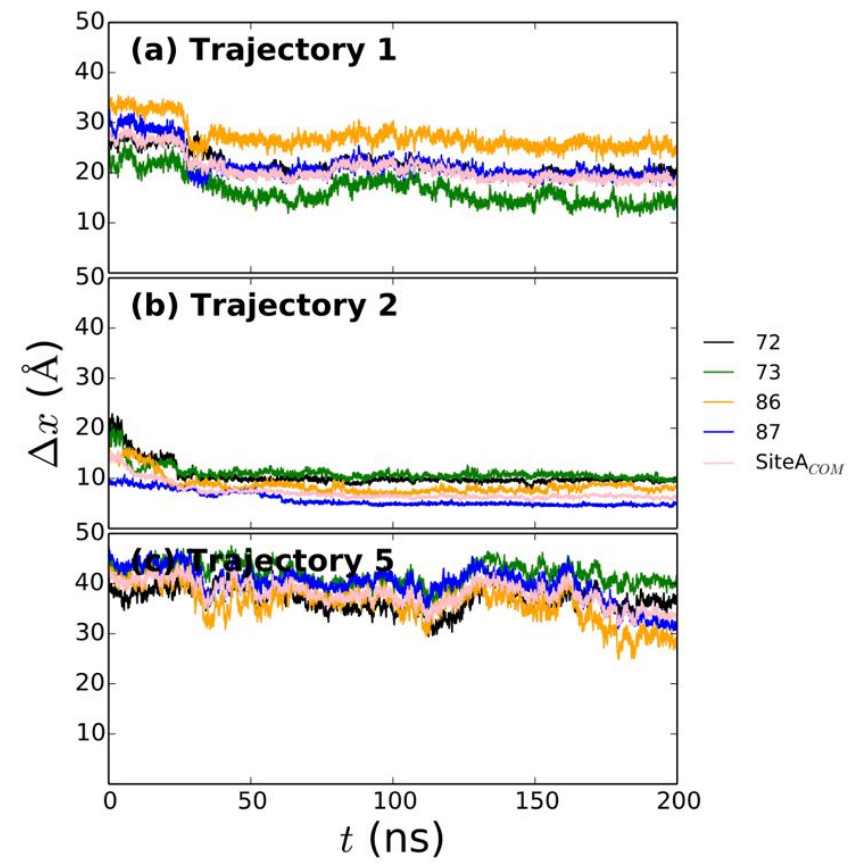

Figure S1.5. The distance $\Delta x$ between the specific groups of amino acidsresidues $\mathrm{K} 72$, $\mathrm{K} 73$, $\mathrm{K} 86$, and $\mathrm{K} 87$, and the $\mathrm{COM}$ of site $\mathrm{A}-$ in the anionic phospholipid binding site A of cyt $c$ and the surface of the MPA-AuNP for trajectories 1,2 , and 5 .

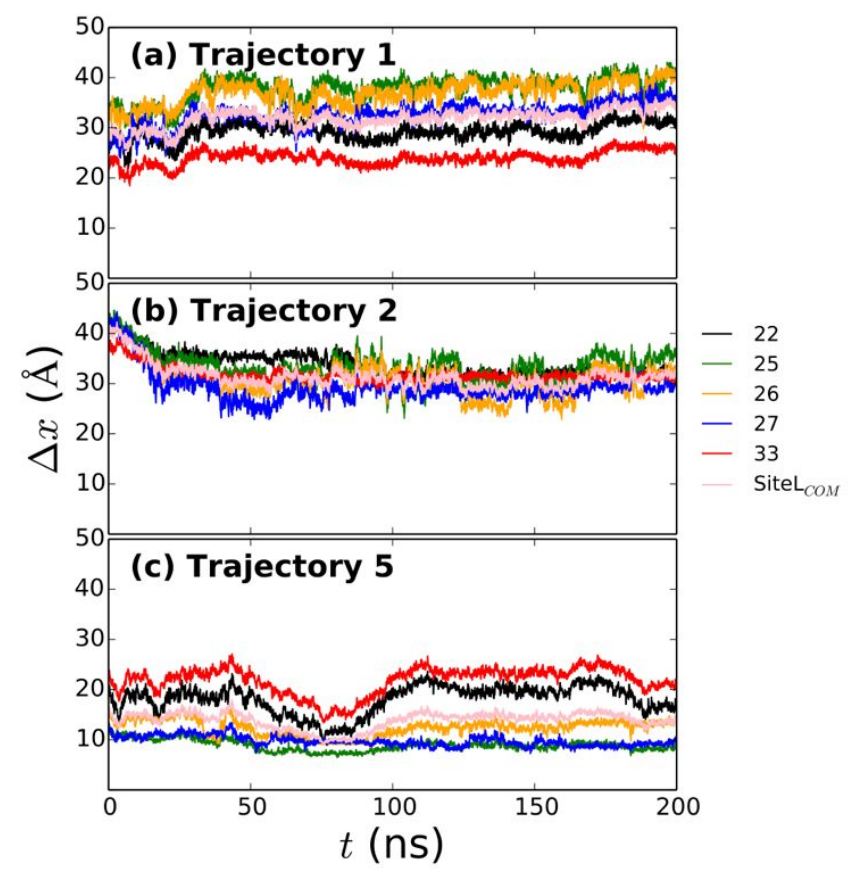

Figure S1.6. The distance $\Delta x$ between the specific groups of amino acids-residues $\mathrm{K} 22$, K25, K26, K27 and K33, and the COM of site L-in the anionic phospholipid binding site $\mathrm{L}$ of cyt $c$ and the surface of the MPA-AuNP for trajectories 1, 2, and 5 . 


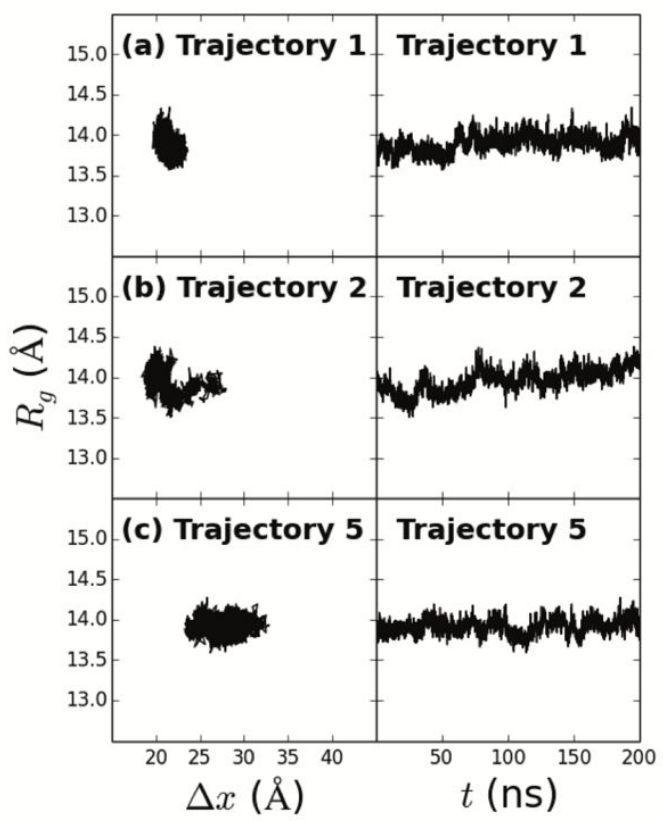

Figure S1.7. The radius of gyration $R_{g}$ of cyt $c$ vs. time along each $200 \mathrm{~ns}$ MD trajectory (right panels) as a function of the distance $\Delta x$ between the cyt $c$ COM and the surface of the MPA-AuNP (left panels).

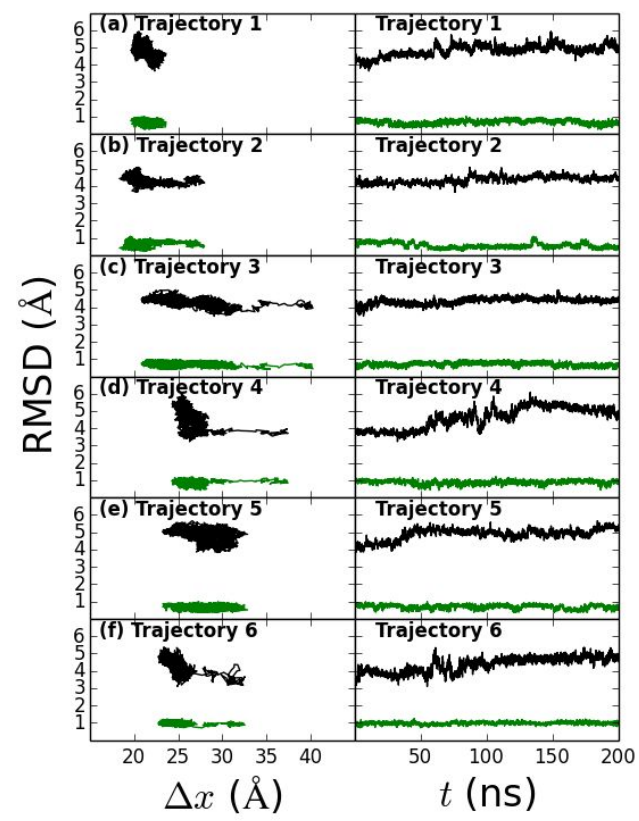

Figure S1.8. The RMSD of cyt $c$ (black) and its heme group (green) vs. time along each $200 \mathrm{~ns}$ MD trajectory (right panels) is also shown as a function of the distance $\Delta x$ between the cyt $c$ COM and the surface of the MPA-AuNP (left panels). In all cases, the RMSD of cyt $c$ is aligned to the equilibrated structure from MD simulations of cyt $c$ in a box of TIP3P for $200 \mathrm{~ns}$. 


\section{Experimental Supplemental Information}

2A. MPA-AuNPs Characterization. MPA-AuNPs were characterized by UV-vis spectroscopy, transmission electron microscopy (TEM), dynamic light scattering measurement (DLS), and $\zeta$ potential analysis. UV-vis spectrum was obtained on a Cary 500 UV-vis-NIR spectrophotometer. Transmission electron microscopy data were obtained with a JEOL 2100 cryo electron microscope operating at $200 \mathrm{kV}$. For TEM imaging, $4.5 \mu \mathrm{L}$ of a dilute solution of AuNPs was drop cast onto a TEM grid (Ted Pella), and the AuNPs sample images were taken with a JEOL 2100 TEM. DLS and $\zeta$-potential measurements were obtained by Malvern Zetasizer Nano-ZS.

A representative UV-vis spectrum of MPA-AuNPs was shown in Fig. S2.1. The diameter of MPAAuNP was estimated in solution by UV-vis spectroscopy to be around $4.4 \mathrm{~nm} .{ }^{2}$ TEM images analysis indicated that the MPA-AuNPs possessed a core diameter of $4.5 \pm 1.4 \mathrm{~nm}(n>200)$. A representative TEM image is shown in Fig. S2.2, and the histogram of size analysis was shown in Fig. S2.3. A representative hydrodynamic size of MPA-AuNPs in $20 \mathrm{mM} \mathrm{NH}_{4} \mathrm{HCO}_{3}$ buffer was $19.6 \pm 0.3 \mathrm{~nm}$, and representative $\zeta$-potential of the MPA-AuNPs $20 \mathrm{mM} \mathrm{NH}_{4} \mathrm{HCO}_{3}$ buffer was $35.9 \pm 8.5 \mathrm{mV}$.

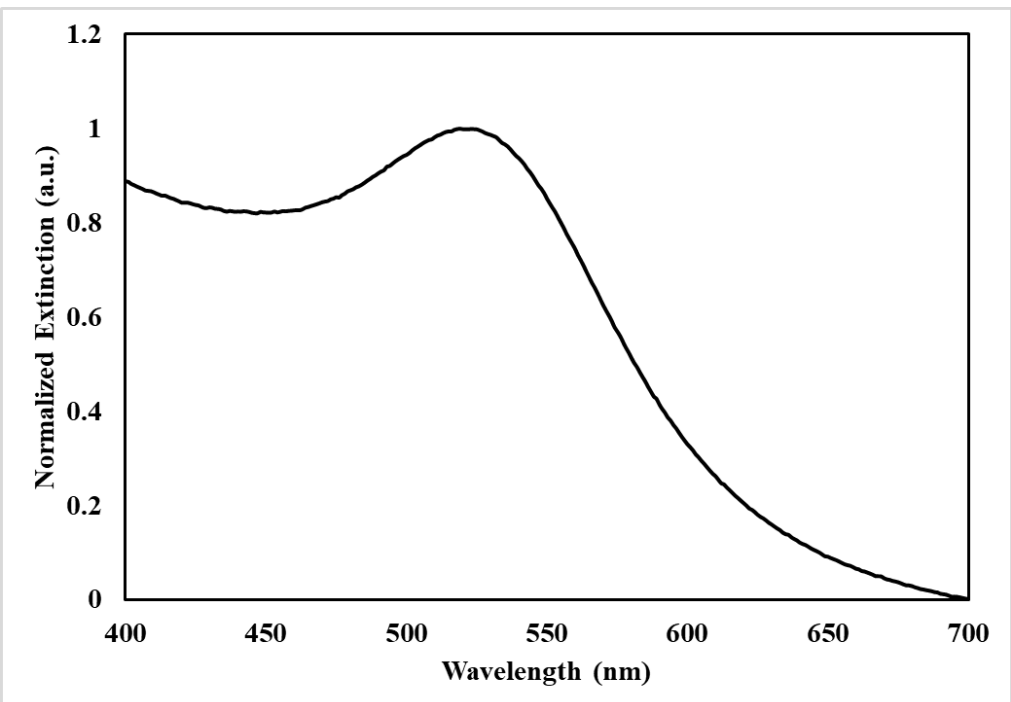

Figure S2.1. Representative UV-vis spectrum of MPA-AuNPs

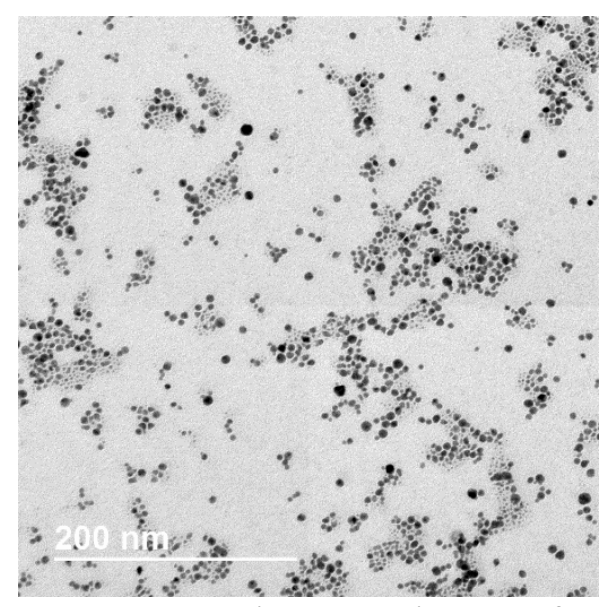

Figure S2.2. Representative TEM image of MPA-AuNPs 


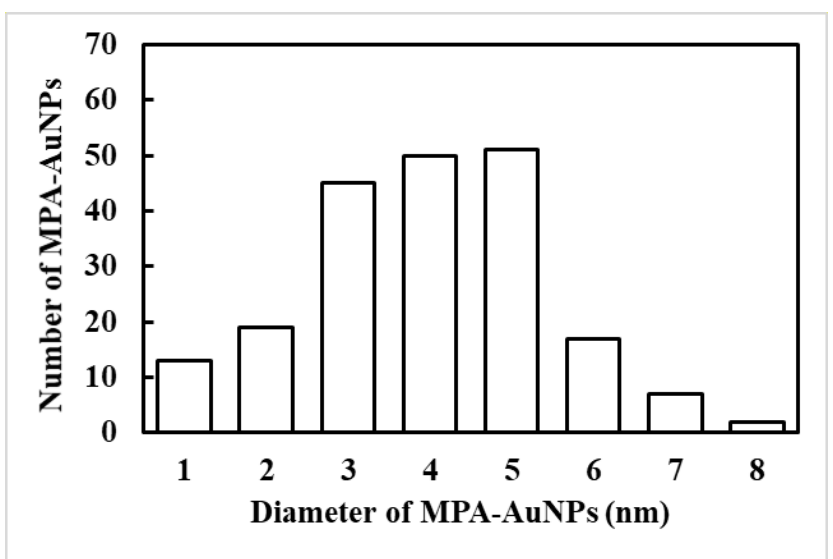

Figure S2.3. Histogram of MPA-AuNPs core diameters as determined by analysis of TEM images.

2B. Protein Adsorption to MPA-AuNPs. UV-vis spectrophotometry and DLS analysis were used for the investigation of adsorption of cyt $c$ to MPA-AuNPs. UV-vis spectrometer is chosen to monitor the localized surface plasmon resonance (LSPR) shifts of AuNPs upon protein adsorption due to the resulting changes in refractive index. ${ }^{3,4}$ The hydrodynamic size of AuNPs increases as the adsorption of protein, which can be measured by DLS.

First, in order to determine the equilibrium time of AuNPs and protein interaction before titration assay, we used UV-vis spectrometer to conduct kinetics experiment, monitoring the plasmon shifts of MPA-AuNPs and cyt $c$ interaction under a series of molar ratios. Figure 4a-g shows the time dependent LSPR peak shifts at different ratio of MPA-AuNPs and cyt $c$. The data point at Time "0" is the wavelength of peak in UV-vis spectrum of $10 \mathrm{nM}$ MPA-AuNPs without addition of cyt $c$. Upon addition of cyt $c$, a kinetics mode of UV-vis spectrometer scanned the wavelength from $550-500 \mathrm{~nm}$ for the sample during $75 \mathrm{~min}$ with a scan rate of $60 \mathrm{~nm} / \mathrm{min}$. As shown, the LSPR peak increased as time goes by, and reached to a plateau after a period depending on the ratio of MPA-AuNPs and cyt $c$. For low concentration of cyt $c$ added (eg. Fig. S2.4a and b), the reaction took longer to reach to an equilibrium (more than $80 \mathrm{~min}$ ), while with a ratio of 1:20 MPA-AuNPs: cyt $c$ (Fig. S2.4d) or furthermore cyt $c$ (Fig. S2.4e-g), it took no more than $20 \mathrm{~min}$ to reach to the plateau. Based on this information, we designed the titration experiment accordingly. 

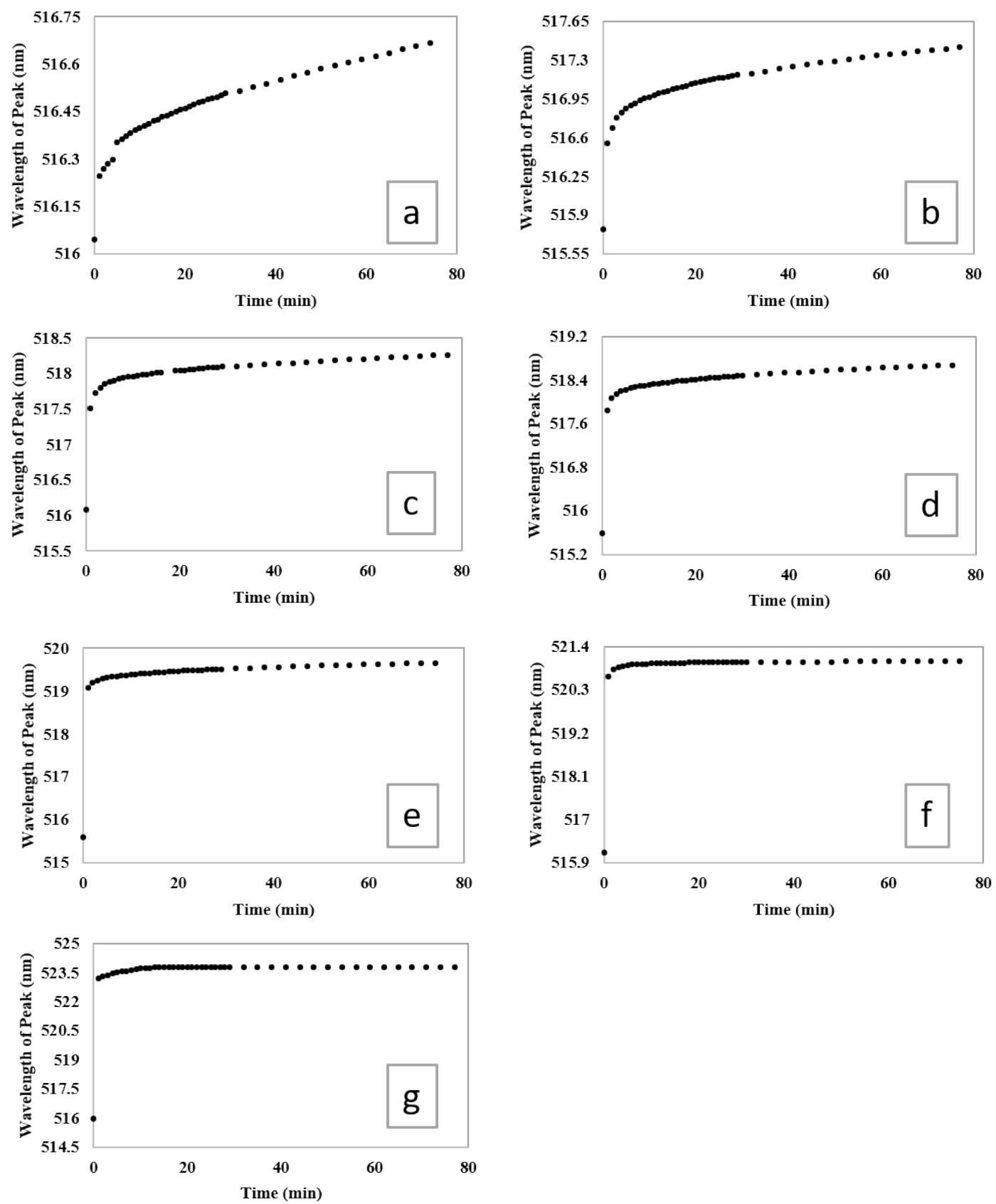

Figure S2.4. Kinetics experiments of MPA-AuNPs and cyt $c$ interaction at MPAAuNPs: cyt $c$ ratio of a) 1:1, b) 1:5, c) 1:10, d) 1:20, e) 1:40, f) 1:100, g) 1:1000. The concentration of MPA-AuNPs was constant (10 nM) and the concentration of cyt $c$ varied to achieve the desired ratios.

A preliminary experiment result confirmed the binding of cyt $c$ to MPA-AuNPs via DLS measurements, which shows an increase of about $4.6 \mathrm{~nm}$ in the hydrodynamic diameter upon addition of $1428.6 \mathrm{nM}$ cyt $c$ to $10 \mathrm{nM}$ MPA-AuNPs, suggesting monolayer adsorption of cyt $c$. Therefore, the binding constant can be estimated by modelling the isotherms with a Langmuir adsorption isotherm: $\Delta / \Delta_{\max }=K_{\mathrm{a}} \times C_{\mathrm{p}} /\left(1+K_{\mathrm{a}} \times \mathrm{C}_{\mathrm{p}}\right)$, where $\Delta$ and $\Delta_{\max }$ are the shift and maximum 
shift in plasmon peak position by UV-vis spectrometry, or the increase and maximum increase in hydrodynamic diameter increase. $K_{\mathrm{a}}$ is the binding constant and $C_{\mathrm{p}}$ is cyt $c$ concentration. ${ }^{3,5}$ Due to the aggregation state of AuNPs, titration data points after $1500 \mathrm{nM}$ cyt $c$ were not used for analysis. The results of titrations are analyzed and shown in Fig. S2.5.

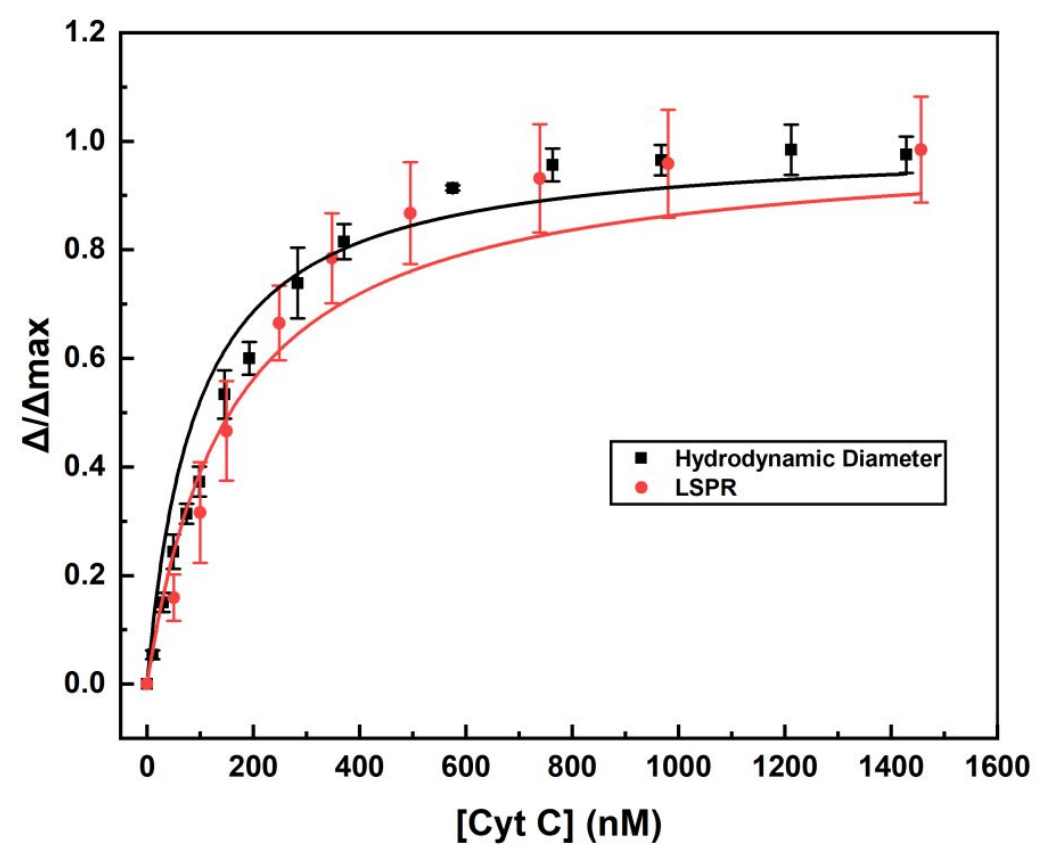

Figure S2.5. $\Delta / \Delta_{\max }$ with respect to cyt $c$ concentration (nM) as cyt $c$ solutions are titrated into $10 \mathrm{nM}$ MPA-AuNPs in $20 \mathrm{mM} \mathrm{NH}_{4} \mathrm{HCO}_{3}$. The data were modeled with the Langmuir adsorption isotherm shown as solid lines (red corresponds to LSPR shift, and black corresponds to hydrodynamic diameter shift). Error bars represent the standard deviation of three replicates.

After fitting with the Langmuir adsorption, the binding constant determined by UV-vis spectrometry is $(6.4 \pm 0.4) \times 10^{6} \mathrm{M}^{-1}$, and the binding constant by DLS measurements is $(10.9 \pm$ 1.4) $\times 10^{6} \mathrm{M}^{-1}$. Based on the models of the LSPR shift, when $\Delta / \Delta_{\max }$ is equal to $0.5(50 \%$ saturation), there are $15 \mathrm{Cyt} C$ per particle; based on the hydrodynamic diameter shift, when $\Delta / \Delta_{\max }$ is equal to 0.5 (50\% saturation), there are $9 \mathrm{Cyt} C$ per particle. Binding constants determined by two complementary methods are in a good agreement.

A colorimetric titration was utilized to determine the protein concentration at which monolayer coverage of MPA-AuNP was achieved (Fig. S2.6). MPA-AuNPs were incubated with increasing concentrations of cyt $c$ for $24 \mathrm{~h}$ before the addition of $1 \mathrm{X}$ PBS, which causes aggregation of nanoparticles not protected by a monolayer of protein. Results indicated the monolayer coverage is achieved between 20 and 40:1 cyt $c$ to MPA-AuNP. 


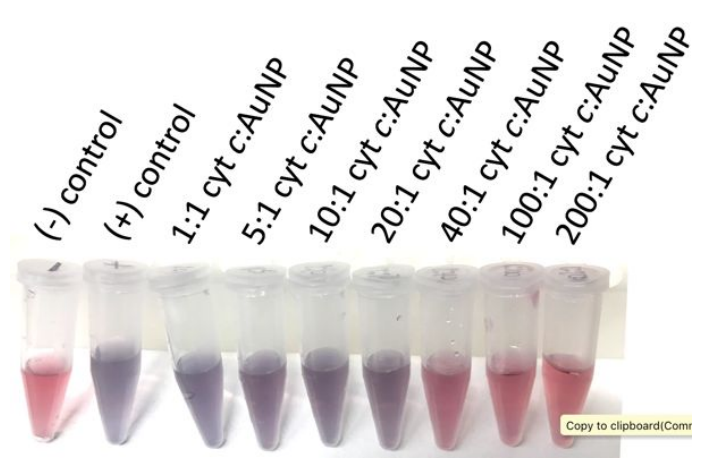

Figure S2.6. A colorimetric titration of cyt $c$ and MPA-AuNP. From left to right: a negative control of MPA-AuNP without PBS induced aggregation (red); a positive control of MPA-AuNP with PBS induced aggregation (purple); 1:1 cyt $c$ :MPAAuNP; 5:1 cyt $c$ :MPA-AuNP; 10:1 cyt $c$ :MPA-AuNP; 20:1 cyt $c$ :MPA-AuNP; 40:1 cyt $c$ :MPA-AuNP; 100:1 cyt $c$ :MPA-AuNP; 200:1 cyt $c$ :MPA-AuNP.

2C. Circular Dichroism. Cyt $c$ adsorbed to MPA-AuNP at $\mathrm{pH} 7.0$ and $\mathrm{pH} 8.6$ were compared by CD. Protein solutions were prepared at $2 \mu \mathrm{M}$ in MilliQ water at either $\mathrm{pH} 7.0$ or 8.6 (adjusted with $0.1 \mathrm{M} \mathrm{NaOH}$ ). MPA-AuNPs were added to each solution for a final AuNP concentration of 180 $\mathrm{nM}$, resulting in a 20:1 cyt $c$ :AuNP ratio, and incubated overnight at $4{ }^{\circ} \mathrm{C}$ before measurements were taken, with the $\mathrm{pH}$ closely monitored. CD spectra were acquired on a JASCO J-815 Spectropolarimeter (JASCO Corporation, Tokyo, Japan) equipped with a temperature regulator. Approximately $250 \mu \mathrm{L}$ of solution in $1 \mathrm{~mm}$ quartz cuvettes were measured at $20{ }^{\circ} \mathrm{C}$, a bandwidth of $1.00 \mathrm{~nm}$, a scanning speed of $50 \mathrm{~nm} / \mathrm{min}$, and baseline corrected to either a water blank for cyt $c$ or an MPA-AuNP blank for cyt $c$ - MPA-AuNP conjugates. The CD traces shown in Fig. S2.7 are an average of 10 scans, and an integration time set as a function of photomultiplier voltage.

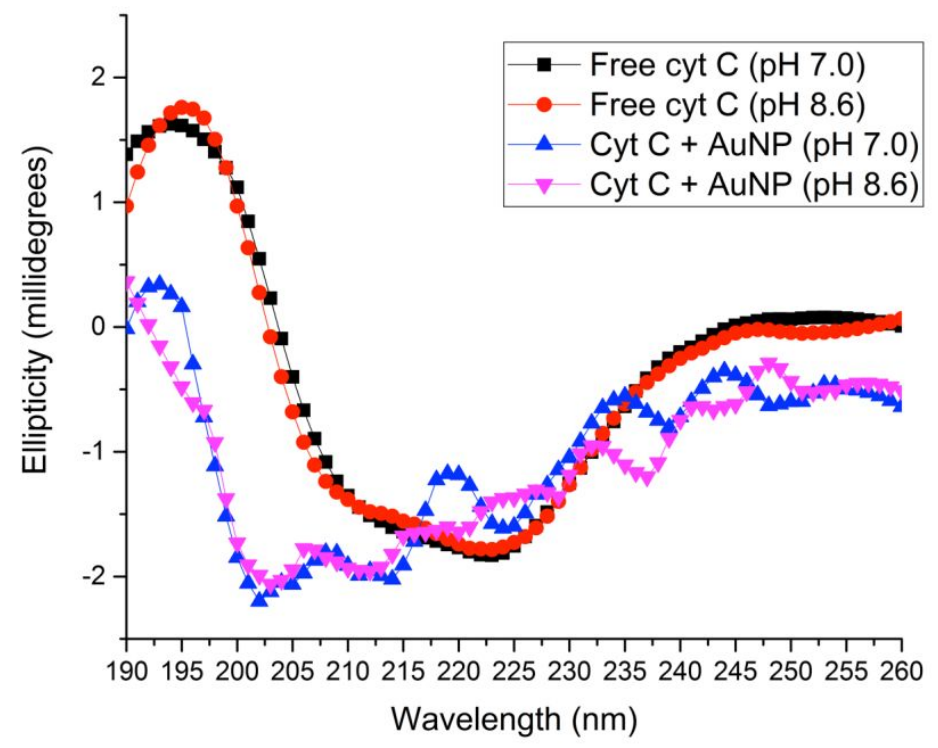

Figure S2.7. Circular dichroism spectra for cyt $c$ free and incubated with MPA AuNPs at $\mathrm{pH} 7.0$ and $\mathrm{pH}$ 8.6.

2D. Synthesis of $N$-acetoxy-succinimide. $N$-acetoxy-succinimide was synthesized according to the procedure reported by Ames and co-workers. ${ }^{6}$ To a $100 \mathrm{~mL}$ round bottom flask was added $N$ - 
hydroxysuccinimide ( $2.9 \mathrm{~g}, 25 \mathrm{mmol}, 1.0$ equiv.) to THF $(25 \mathrm{~mL})$. The solution was set to stir at $4{ }^{\circ} \mathrm{C}$ before acetic anhydride $(2.8 \mathrm{~mL}, 30 \mathrm{mmol}, 1.2$ equiv. $)$ was added dropwise. Then $\mathrm{NEt}_{3}(3.8$ $\mathrm{mL}, 28 \mathrm{mmol}, 1.1$ equiv.) was slowly added and the reaction was stirred at $4{ }^{\circ} \mathrm{C}$ for $30 \mathrm{~min}$. Hexanes $(30 \mathrm{~mL})$ were then added and the reaction was allowed to stand at ambient temperature without stirring for $1 \mathrm{~h}$. The white, solid product was recovered via filtration, dried overnight in vacuo, and stored at $-20{ }^{\circ} \mathrm{C}$ for up to 6 months without degradation. Analytical data is consistent with literature values. ${ }^{7}$ m.p. $132-135^{\circ} \mathrm{C} ;{ }^{1} \mathrm{H}$ NMR $\left(400 \mathrm{MHz}, \mathrm{CDCl}_{3}\right) \delta 2.86(\mathrm{~s}, 4 \mathrm{H}), 2.37(\mathrm{~s}, 3 \mathrm{H})$; ${ }^{13} \mathrm{C}$ NMR (125.7 MHz, $\mathrm{CDCl}_{3}$ ) $\delta 169.1$ (2C), $165.6(1 \mathrm{C}), 25.6$ (2C), $17.6(1 \mathrm{C})$.

2E. MALDI-TOF Analysis and Optimization of Lysine Labeling Conditions. To determine the appropriate labeling reagent equivalences, reactions were carried out at $1,000 \mathrm{X}$ and $10,000 \mathrm{X}$ excess $\mathrm{N}$-acetoxy-succinimide at $\mathrm{pH} 8.6$ and whole protein samples were examined by MALDI TOF (Applied Biosystems-Sciex 5800 MALDI-TOF/TOF Mass Spectrometer) with a sinapic acid matrix (Fig. S2.8). Increased labeling was consistently observed at 10,000× excess compared to $1,000 \times$ excess. Next, the reaction time was compared at $1 \mathrm{~min}$ and $5 \mathrm{~min}$ at $10,000 \times$ excess $\mathrm{N}$ acetoxy-succinimide, and showed no increased labeling between 1 and 5 min (Fig. S2.8). 

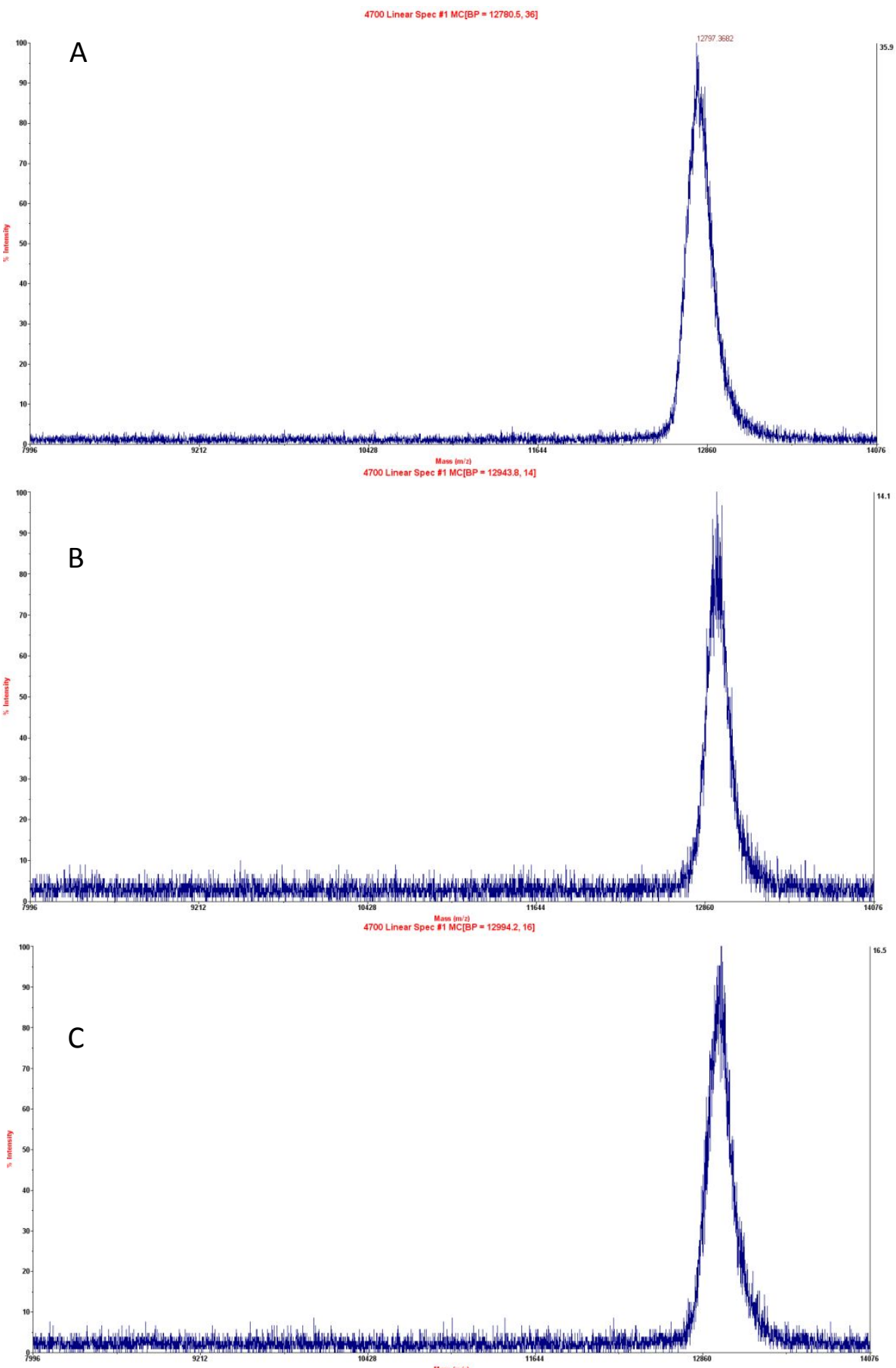

Figure S2.8. MALDI-TOF analysis of labeled cyt $c$ with a) $1,000 \mathrm{X}$ excess $N$ acetoxy-succinimide for $5 \mathrm{~min}$, b) $10,000 \mathrm{X}$ excess $\mathrm{N}$-acetoxy-succinimide for 5 min, and c) $10,000 \mathrm{X}$ excess $N$-acetoxy-succidinimde for $1 \mathrm{~min}$. 
2F. Protein Footprinting Workflow. The protein footprinting workflow is shown in Fig. S2.9. Cyt $c$ is first incubated for $18 \mathrm{~h}$ in the presence of MPA-AuNP (top) or without (bottom). Next, $N$ acetoxy-succinimide is used in 10,000 molar excess of cyt $c$ for lysine labeling for $1 \mathrm{~min}$, at which point excess reagent is quenched with Tris. To recover the entire protein, the MPA-AuNP is dissolved by treatment of the samples with KCN, washing the protein with 10,000 MWC filters, and evaporating them to dryness. Finally, the proteins are digested with chymotrypsin and the resulting peptides are subjected to LC-MS/MS for percent lysine modification analysis and comparison.

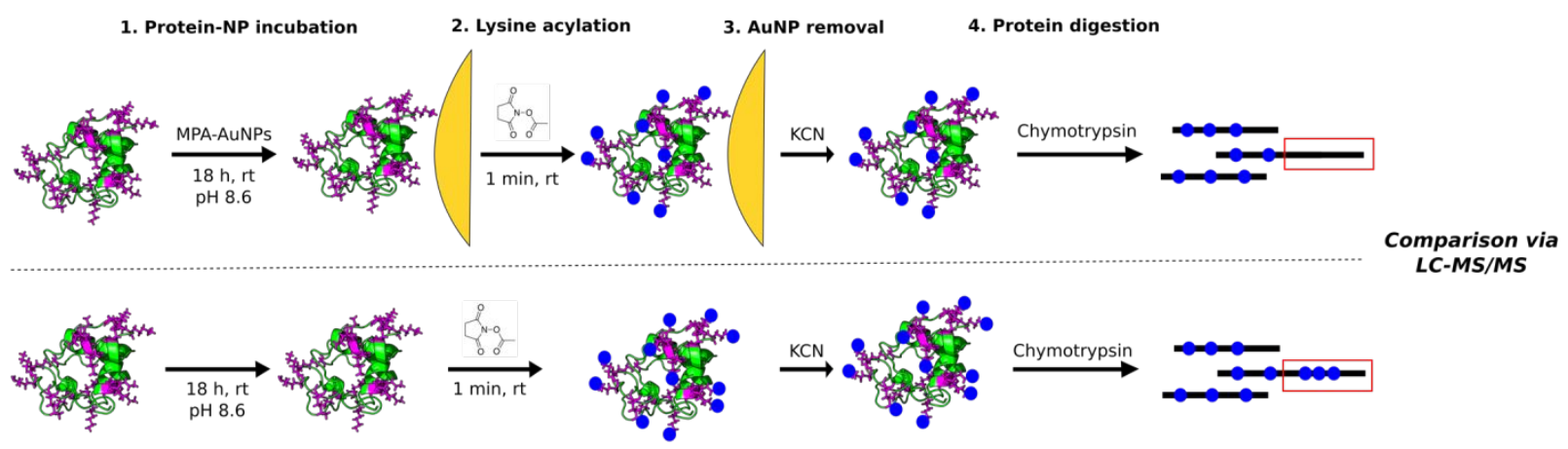

Figure S2.9. Protein footprinting workflow. (Details described in the text of section 2F.)

2G. PEAKS Search Parameters and Summary Table. Peptide identification and percent modification were carried out with PEAKS 8.5 software (BioInformatics Software Inc.) with the search parameters listed in Table S1. For percent modification calculations, only peptides with a $p$ value $\leq 0.01$ and residue modification assignments with a $p$ value $\leq 0.01$ were included in the calculations to ensure confidence in assignment. Statistical analysis of the data was performed with non-parametric one-way ANOVA (Table S2; $\alpha=0.05 ; * * * p<0.001 ; * * p<0.01 ; * p<0.05$ ). 
Table S1. Search parameters used with PEAKS 8.5 for protein footprinting analysis.

\begin{tabular}{|c|c|}
\hline Search Engine Name & PEAKS \\
\hline Parent Mass Error Tolerance & $20.0 \mathrm{ppm}$ \\
\hline Fragment Mass Error Tolerance & $0.1 \mathrm{Da}$ \\
\hline Precursor Mass Search Type & monoisotopic \\
\hline Enzyme & Chymotrypsin \\
\hline Max Missed Cleavages & 2 \\
\hline Non-specific Cleavage & none \\
\hline Variable Modfications & 42.01 \\
\hline Acetylation (K) & 5 \\
\hline Max Variable PTM Per Peptide & {$[$ Uniprot_SwissProt] } \\
\hline Database & Equus caballus (horse) \\
\hline Taxon & 288 \\
\hline Searched Entry & Enabled \\
\hline FDR Estimation & 4 \\
\hline De Novo Dependencies & \\
\hline
\end{tabular}


Table S2. Summary of percent modification of detected lysines.

\begin{tabular}{|c|l|r|r|r|r|r|}
\hline & Peptide Sequence & Cyt C & Std. Dev. & $\begin{array}{r}\text { Cyt C + } \\
\text { MPA } \\
\text { AuNP }\end{array}$ & Std. Dev. & p \\
\hline K5 & $* * * *$ MGDVEkGKKIFVQKCA & $68.6 \%$ & $16.4 \%$ & $92.7 \%$ & $2.8 \%$ & $3.17 \mathrm{E}-03$ \\
\hline K7 & $* * *$ MGDVEKGkKIFVQKCAQC & $51.1 \%$ & $26.1 \%$ & $56.9 \%$ & $32.9 \%$ & $7.55 \mathrm{E}-01$ \\
\hline K8 & $* *$ MGDVEKGKkIFVQKCAQCH & $55.3 \%$ & $15.6 \%$ & $20.5 \%$ & $23.0 \%$ & $2.13 \mathrm{E}-01$ \\
\hline K22 & QKCAQCHTVEkGGKHKTGPNL & $100.0 \%$ & $0.1 \%$ & $71.1 \%$ & $14.2 \%$ & $2.39 \mathrm{E}-04$ \\
\hline K25 & AQCHTVEKGGkHKTGPNLHGL & $87.9 \%$ & $4.1 \%$ & $71.5 \%$ & $6.5 \%$ & $2.70 \mathrm{E}-05$ \\
\hline K27 & CHTVEKGGKHkTGPNLHGLFG & $75.8 \%$ & $4.0 \%$ & $61.7 \%$ & $9.1 \%$ & $1.26 \mathrm{E}-03$ \\
\hline K39 & GPNLHGLFGRkTGQAPGFTYT & $38.7 \%$ & $9.7 \%$ & $66.6 \%$ & $3.7 \%$ & $3.00 \mathrm{E}-06$ \\
\hline K72 & ETLMEYLENPkKYIPGTKMIF & $53.6 \%$ & $18.8 \%$ & $84.2 \%$ & $8.8 \%$ & $1.27 \mathrm{E}-03$ \\
\hline K73 & TLMEYLENPKkYIPGTKMIFA & $55.0 \%$ & $14.1 \%$ & $82.7 \%$ & $6.9 \%$ & $2.72 \mathrm{E}-04$ \\
\hline K79 & ENPKKYIPGTkMIFAGIKKKT & $84.3 \%$ & $4.4 \%$ & $71.7 \%$ & $4.5 \%$ & $6.08 \mathrm{E}-05$ \\
\hline K86 & PGTKMIFAGIkKKTEREDLIA & $76.3 \%$ & $5.5 \%$ & $68.8 \%$ & $6.7 \%$ & $3.00 \mathrm{E}-02$ \\
\hline K87 & GTKMIFAGIKkKTEREDLIAY & $57.2 \%$ & $10.9 \%$ & $54.6 \%$ & $7.9 \%$ & $6.07 \mathrm{E}-01$ \\
\hline K88 & TKMIFAGIKKkTEREDLIAYL & $52.1 \%$ & $6.9 \%$ & $56.9 \%$ & $7.6 \%$ & $2.20 \mathrm{E}-01$ \\
\hline K99 & TEREDLIAYLkKATNE***** & $94.8 \%$ & $6.8 \%$ & $98.8 \%$ & $1.9 \%$ & $1.56 \mathrm{E}-01$ \\
\hline K100 & EREDLIAYLKkATNE****** & $89.2 \%$ & $8.9 \%$ & $85.2 \%$ & $16.2 \%$ & $5.51 \mathrm{E}-01$ \\
\hline
\end{tabular}

\section{Additional Files}

LC-MS/MS was performed on a Thermo Scientific Orbitrap Elite and a Luna C18 column (Phenomenex; $75 \mu \mathrm{m}$ inner diameter, $20 \mathrm{~cm}$ length, $100 \AA$ pore size). All MS measurements were performed in the positive ion mode via higher-energy collisional dissociation (HCD). Precursor ions were measured at a resolution of 60,000 in the Orbitrap analyzer. The top ten most intense ions from each MS scan were chosen for isolation, fragmentation, and detection. The experimental .raw files (LC-MS/MS .raw files) are available upon request as a $3.5 \mathrm{~GB}$ zip file. The spreadsheet used to analyze percent modification (EJT-III-092 series analysis.xlsx) is available for download as part of the SI associated with this article online. 
Table S3. Detailed listing of the .raw files from the experiments.

\begin{tabular}{|c|c|}
\hline File Name & Data Set Description \\
\hline EJT-III-092Ai92Ai.raw & $\begin{array}{l}\text { Cyt } c+\text { MPA-AuNP biological replicate } 1 ; 1^{\text {st }} \\
\text { technical replicate }\end{array}$ \\
\hline EJT-III-092Aii92Aii.raw & $\begin{array}{l}\text { Cyt } c+\text { MPA-AuNP biological replicate } 1 ; 2^{\text {nd }} \\
\text { technical replicate }\end{array}$ \\
\hline EJT-III-092Aiii92Aiii.raw & $\begin{array}{l}\text { Cyt } c+\text { MPA-AuNP biological replicate } 1 ; 3^{\text {rd }} \\
\text { technical replicate }\end{array}$ \\
\hline EJT-III-092Bi92Bi.raw & $\begin{array}{l}\text { Cyt } c+\text { MPA-AuNP biological replicate } 2 ; 1^{\text {st }} \\
\text { technical replicate }\end{array}$ \\
\hline EJT-III-092Bii92Bii.raw & $\begin{array}{l}\text { Cyt } c+\text { MPA-AuNP biological replicate } 2 ; 2^{\text {nd }} \\
\text { technical replicate }\end{array}$ \\
\hline EJT-III-092Biii92Biii.raw & $\begin{array}{l}\text { Cyt } c+\text { MPA-AuNP biological replicate } 2 ; 3^{\text {rd }} \\
\text { technical replicate }\end{array}$ \\
\hline EJT-III-092Ci92Ci.raw & $\begin{array}{l}\text { Cyt } c+\text { MPA-AuNP biological replicate } 3 ; 1^{\text {st }} \\
\text { technical replicate }\end{array}$ \\
\hline EJT-III-092Cii92Cii.raw & $\begin{array}{l}\text { Cyt } c+\text { MPA-AuNP biological replicate } 3 ; 2^{\text {nd }} \\
\text { technical replicate }\end{array}$ \\
\hline EJT-III-092Ciii92Dii.raw & $\begin{array}{l}\text { Cyt } c+\text { MPA-AuNP biological replicate } 3 ; 3^{\text {rd }} \\
\text { technical replicate }\end{array}$ \\
\hline EJT-III-092Di92Diii.raw & Cyt $c$ biological replicate $1 ; 1^{\text {st }}$ technical replicate \\
\hline EJT-III-092Dii92Ei.raw & Cyt $c$ biological replicate $1 ; 2^{\text {nd }}$ technical replicate \\
\hline EJT-III-092Diii92Eii.raw & Cyt $c$ biological replicate $1 ; 3^{\text {rd }}$ technical replicate \\
\hline EJT-III-092Ei92Eiii.raw & Cyt $c$ biological replicate $2 ; 1^{\text {st }}$ technical replicate \\
\hline EJT-III-092Eii92Fi.raw & Cyt $c$ biological replicate $2 ; 2^{\text {nd }}$ technical replicate \\
\hline EJT-III-092Eiii92Fii.raw & Cyt $c$ biological replicate $2 ; 3^{\text {rd }}$ technical replicate \\
\hline EJT-III-092Fi92Fiii.raw & Cyt $c$ biological replicate 3 ; $1^{\text {st }}$ technical replicate \\
\hline EJT-III-092Fii92ciii.raw & Cyt $c$ biological replicate $3 ; 2^{\text {nd }}$ technical replicate \\
\hline EJT-III-092Fiii92di.raw & Cyt $c$ biological replicate $3 ; 3^{\text {rd }}$ technical replicate \\
\hline EJT-III-092 series analysis & $\begin{array}{l}\text { Spreadsheet with percent modification exports from } \\
\text { PEAKS PTM and statistical analysis }\end{array}$ \\
\hline
\end{tabular}

\section{Reference:}

1. Kataoka, M.; Hagihara, Y.; Mihara, K.; Goto, Y. Molten Globule of Cytochrome-C Studied by Small-Angle X-Ray-Scattering. J. Mol. Biol. 1993, 229, 591-596.

2. Haiss, W.; Thanh, N. T. K.; Aveyard, J.; Fernig, D. G. Determination of Size and Concentration of Gold Nanoparticles from UV-Vis Spectra. Anal Chem 2007, 79, 4215-4221. 3. Dennison, J. M.; Zupancic, J. M.; Lin, W.; Dwyer, J. H.; Murphy, C. J. Protein Adsorption to Charged Gold Nanospheres as a Function of Protein Deformability. Langmuir 2017, 33, 7751-7761. 
4. Willets, K. A.; Duyne, R. P. V. Localized Surface Plasmon Resonance Spectroscopy and Sensing. Annu. Rev. Phys. Chem. 2007, 58, 267-297.

5. Yang, J. A.; Johnson, B. J.; Wu, S.; Woods, W. S.; George, J. M.; Murphy, C. J. Study of Wild-Type $\alpha$-Synuclein Binding and Orientation on Gold Nanoparticles. Langmuir 2013, 29, 4603-4615.

6. Ames, D. E.; Grey, T. F. The Synthesis of Some N-Hydroxyimides. J. Chem. Soc. 1955, 631-636. 